\title{
Von der Hierarchie zum Team? Interprofessionalität im Schweizer Gesundheitswesen
}

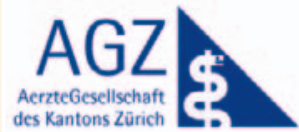

Universität

Zürich ${ }^{\text {uzt }}$

Medizinhistorisches Institut und Museum

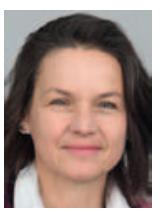

Christina Brunnschweiler

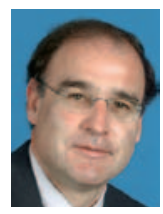

Pierre-Alain Clavien

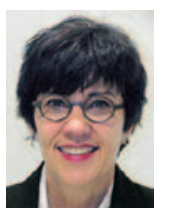

Gabriella Chiesa

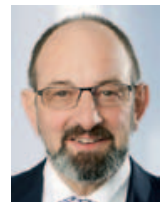

Ernst Gähler

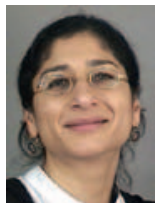

Maya Shaha

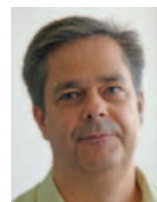

Eberhard Wolff

* www.pflegeportal.ch/ pflegeportal/pub/zusammen arbeit aerztlich nichtaerzlich_866_1.pdf

** www.mhiz.uzh.ch/ departments/Museum/ WhoCares.htm
Gesamtgesellschaftliche, aber auch spezifische Entwicklungen im Gesundheitssektor stellen seit einigen Jahren und insbesondere in Zukunft neue Anforderungen an die Zusammenarbeit der im Medizinalbereich tätigen Berufsgruppen. Stichworte dazu sind «demographische Entwicklung», «Zunahme chronischer Krankheiten» oder «Ärztemangel». Dass die Kooperation zwischen den unterschiedlichen Berufsgruppen verbessert werden soll, um eine bessere Versorgung kranker Menschen zu ermöglichen, ist weitgehend unbestritten. Wenn es dagegen um die konkrete Ausgestaltung der Zusammenarbeit, um Rollenbilder, Zuständigkeiten und Kompetenzen geht, sind beträchtliche Divergenzen zu beobachten.

\section{Diskutieren Sie mit}

Wo liegen die aktuellen Stärken und Schwächen der interprofessionellen Zusammenarbeit in unserem Gesundheitssystem? Welche neuen Formen der Ko-

\section{Die Podiumsgäste}

Es diskutieren unter der Leitung von SÄZ-Redaktor PD Dr. rer. soc. Eberhard Wolff, Beauftragter Sonderausstellungen, Medizinhistorisches Museum der Universität Zürich:

- Christina Brunnschweiler, lic. oec. HSG, CEO Spitex Zürich Limmat

- Prof. Dr. med. Pierre-Alain Clavien, Direktor Klinik für Viszeral- und Transplantationschirurgie, UniversitätsSpital Zürich

Die Veranstaltung wird organisiert in Zusammenarbeit mit der Ärztegesellschaft des Kantons Zürich und dem Medizinhistorischen Institut und Museum der Universität Zürich.

\section{Eintritt frei - Anmeldung erforderlich}

Die öffentliche Podiumsdiskussion mit anschliessendem Apéro findet statt am Donnerstag, 19. April 2012, 18.15-20 Uhr im Gebäude des Medizinhistorischen Museums, Rämistrasse 69, Zürich (Hörsaal SOC 106). Der Eintritt ist frei, eine Anmeldung ist aber erforderlich. Diese kann bis Dienstag, 17. April via E-Mail an redaktion.saez[at]emh.ch oder via Fax an 0614678556 erfolgen. Bitte Ihren Namen und die Namen allfälliger Begleitpersonen sowie das Stichwort «Anmeldung zum SÄZ-Podium vom 19. April» angeben. Auch telefonische Anmeldungen sind vormittags unter 0614678572 möglich. operation sind gefragt, um den zukünftigen Herausforderungen wirksam zu begegnen? Krankt unser Gesundheitswesen tatsächlich an einer zu «ausgeprägten Arztzentriertheit», soll «jedwede Hierarchie aus der Gesundheitsversorgung entfernt werden», wie in einem deutschen Grundsatzpapier* diagnostiziert bzw. gefordert wird?

Der Einbezug des Publikums in die Diskussion ist zentraler Bestandteil des Konzepts der SÄZ-Podien, mit denen ein interessanter und konstruktiver Beitrag zur Debatte aktueller gesundheitspolitischer Fragen

Hintergrund der Veranstaltung ist die Wanderausstellung «WHO CARES» im Medizinhistorischen Museum der Universität Zürich, die sich mit der Geschichte und Gegenwart des Pflegeberufs in Deutschland befasst. **

Vor der Podiumsdiskussion findet um 17 Uhr eine Sonderführung mit Institiutsdirektor Prof. Flurin Condrau durch die Ausstellung statt.

- Gabriella Chiesa, eidg. dipl. pharm., Leiterin Innovation Versorgungsmanagement, CSS Versicherung

- Dr. med. Ernst Gähler, Vizepräsident der FMH,

- Maya Shaha, PhD, RN, wissenschaftliche Mitarbeiterin, Direktion Pflege, MTT Inselspital Bern, MER, Institut universitaire de formation et de recherche en soins, CHUV Lausanne.

Die Durchführung des Anlasses wird möglich dank grosszügiger Unterstützung durch Interpharma, den Verband der forschenden pharmazeutischen Industrie. Die Verantwortung für Konzept und Inhalt des Podiums liegt bei der Schweizerischen Ärztezeitung. geleistet werden soll. Facharzt für Allgemeinmedizin FMH 